\title{
Surface Terms of Quartic Quasitopological Gravity and Thermodynamics of Nonlinear Charged Rotating Black Branes
}

\author{
A. Bazrafshan ${ }^{1}$, M. H. Dehghani ${ }^{2,3}{ }^{*}$, and M. Ghanaatian ${ }^{4}$ \\ 1 Department of Physics, Jahrom University, 74137-66171 Jahrom, Iran \\ 2 Center for Excellence in Astronomy \& Astrophysics (CEAA \\ - RIAAM) - Maragha, IRAN, P. O. Box: $55134-441$ \\ ${ }^{3}$ Physics Department and Biruni Observatory, \\ College of Sciences, Shiraz University, Shiraz 71454, Iran and \\ 4 Department of Physics, Payame Noor University, Iran
}

\begin{abstract}
As in the case of Einstein or Lovelock gravity, the action of quartic quasitopological gravity has not a well-defined variational principle. In this paper, we first introduce a surface term that makes the variation of quartic quasitopological gravity well defined. Second, we present the static charged solutions of quartic quasitopological gravity in the presence of a non linear electromagnetic field. One of the branch of these solutions presents a black brane with one or two horizons or a naked singularity depending on the charge and mass of the solution. The thermodynamic of these black branes are investigated through the use of the Gibbs free energy. In order to do this, we calculate the finite action by use of the counterterm method inspired by AdS/CFT correspondence. Introducing a Smarr-type formula, we also show that the conserved and thermodynamics quantities of these solutions satisfy the first law of thermodynamics. Finally, we present the charged rotating black branes in $(n+1)$ dimensions with $k \leq[n / 2]$ rotation parameters and investigate their thermodynamics.
\end{abstract}

* email address: mhd@shirazu.ac.ir 


\section{INTRODUCTION}

The anti-de Sitter/conformal field theory (AdS/CFT) correspondence [1] provides a prescription to compute vacuum expectation values of the $n$-dimensional conformal field theory (CFT) operators in terms of dual classical field in an $(n+1)$-dimensional anti-de Sitter (AdS) spacetime. This prescription has been checked successfully in many examples. It is now known that the central charges in the $n$-dimensional conformal field theory relate to the coupling constants of the $(n+1)$-dimensional gravity. But, since Einstein gravity has only one coupling constant, it is only dual to those conformal field theories for which all the central charges are equal. In order to extend the duality to new classes of conformal field theories with different ratios between central charges, one should consider theories of gravity with enough parameters to account for the ratios between central charges. In this case, one should add higher curvature terms with more coupling constants within a perturbative framework to action [2]. The computation of central charges and their relation with the coupling constants of Lovelock gravity [3] have been investigated for the case of second and third order Lovelock gravity [4, 5]. Lovelock gravity is the most general theory of gravitation which is quasilinear in the second derivatives of the metric and does not contain any higher derivatives in any dimension for a general spacetime. Although the equations of motion of $n$th order Lovelock gravity are second-order differential equations, the $n$th order Lovelock term has no contribution to the field equations in $2 n$ and lower dimensions and therefore it is useless for studying field theories in four dimensions. In order to have gravitational theory in five dimension with more coupling constants, one should study higher derivative gravity or quasitopological gravity. Although the field equations of higher derivative grav-

ity contain higher derivatives of the metric more than two, recently, third [6] and fourth [7] order quasitopological terms have been introduced which have contribution to the field equations in five and higher dimensions, and the equations of motion of these theories are second-order differential equations when the metric is spherically symmetry. As in the case of third and fourth order Lovelock gravities, the cubic and quartic terms have no contribution to the field equations in six and eight dimensions, respectively. Although this property of Lovelock gravity is due to a topological origin of Euler density, this property of the new theory does not have a topological origin and therefore these theories are known as cubic and quartic quasitopological gravity. The black hole solutions and holography study of cubic 
quasitopological gravities have been investigated in Refs. [8 10].

The main aim of this paper is to introduce a surface term for quartic quasitopological gravity introduced in [7] in order to have a well-defined variational principle in the case of spacetimes with flat boundary. As for the case of the Einstein-Hilbert action which does not have a well-defined variational principle [11], the variation of the action of quasitopological gravity is not well-defined. This is due to the fact that one encounters a total derivative that produces a surface integral involving the derivative of the variation of the metric normal to the boundary. To cancel this normal derivative term, one has to add a surface term to the action. The surface terms of Lovelock and cubic quasitopological gravity have been introduced in [12, 13] and [14], respectively. Here, we introduce the surface term for quartic quasitopological gravity. Another motivation of introducing the surfaceterm is due to the fact that in the Hamiltonian formalism of general relativity, the surface terms should be known [15].

Our second goal is to introduce the black brane solutions of quartic quasitopological gravity in the presence of a nonlinear Maxwell field. Having nonlinear terms of curvature in the action of gravity, it is natural to assume nonlinear terms of electromagnetic tensor field, $\left(-F_{\mu \nu} F^{\mu \nu}\right)^{s}$, in the matter action [16]. Our third aim is to introduce a counterterm that removes the divergences of the action and conserved quantities of the black brane solutions. Finally, we investigate the thermodynamics of static and rotating black brane solutions of quartic quasitopological gravity in the presence of power law Maxwell field.

The outline of our paper is as follows. In Sec. II, we give a brief review of the quartic quasitopological gravity and review the surface terms which make the variation of Einstein, Gauss-Bonnet and cubic quasitopological terms well defined for flat boundary. Then we introduce the surface term of quasitopological gravity for spacetimes with flat boundary. In Sec. III, we introduce asymptotically AdS charged black branes of quasitopological gravity in the presence of nonlinear Maxwell field. Section [V] is devoted to the investigation of the thermodynamic properties of these solutions by using the relation between the on shell action and Gibbs free energy. In Sec. V, we endow our solutions with rotation and study the thermodynamic properties of these rotating charged black branes. Finally, we finish our paper with some concluding remarks. 


\section{SURFACE TERMS OF QUARTIC QUASITOPOLOGICAL GRAVITY}

The field equations of fourth order Lovelock gravity are at most second order partial differential equations, while it contains fourth order terms in Riemann tensor. But, the fourth order term has contribution in the field equations provided one consider the field equations in nine and higher dimensions. On the other hand, quartic quasitopological gravity is a theory of gravity which contains fourth order terms in Riemann tensor with at most second order differential equation, while the quartic term contributes to the field equations in five and higher dimensions [7]. Of course, one should note that the field equations of quartic quasitopological gravity are second order differential equations only for a spherically symmetric spacetime, and has no contribution in eight dimensions. The action of fourth order quasitopological gravity in $(n+1)$ dimensions in the presence of a nonlinear electromagnetic field may be written as

$$
I_{G}=\frac{1}{16 \pi} \int d^{n+1} x \sqrt{-g}\left[-2 \Lambda+\mathcal{L}_{1}+\mu_{2} \mathcal{L}_{2}+\mu_{3} \mathcal{X}_{3}+\mu_{4} \mathcal{X}_{4}+L(F)\right]
$$

where $\Lambda=-n(n-1) / 2 l^{2}$ is the cosmological constant of AdS spacetime, $\mathcal{L}_{1}=R$ is just the Einstein-Hilbert Lagrangian, $\mathcal{L}_{2}=R_{a b c d} R^{a b c d}-4 R_{a b} R^{a b}+R^{2}$ is the second order Lovelock (Gauss-Bonnet) Lagrangian, $\mathcal{X}_{3}$ is the curvature-cubed Lagrangian [4]

$$
\begin{aligned}
\mathcal{X}_{3}= & R_{a b}^{c d} R_{c d}^{e}{ }^{f} R_{e f}^{a b}+\frac{1}{(2 n-1)(n-3)}\left(\frac{3(3 n-5)}{8} R_{a b c d} R^{a b c d} R\right. \\
& -3(n-1) R_{a b c d} R^{a b c}{ }_{e} R^{d e}+3(n+1) R_{a b c d} R^{a c} R^{b d} \\
& \left.+6(n-1) R_{a}{ }^{b} R_{b}{ }^{c} R_{c}{ }^{a}-\frac{3(3 n-1)}{2} R_{a}^{b} R_{b}{ }^{a} R+\frac{3(n+1)}{8} R^{3}\right),
\end{aligned}
$$

and $\mathcal{X}_{4}$ is the fourth order term of quasitopological gravity [7]

$$
\begin{aligned}
\mathcal{X}_{4}= & c_{1} R_{a b c d} R^{c d e f} R_{e f}^{h g} R_{h g}^{a b}+c_{2} R_{a b c d} R^{a b c d} R_{e f} R^{e f}+c_{3} R R_{a b} R^{a c} R_{c}{ }^{b}+c_{4}\left(R_{a b c d} R^{a b c d}\right)^{2} \\
& +c_{5} R_{a b} R^{a c} R_{c d} R^{d b}+c_{6} R R_{a b c d} R^{a c} R^{d b}+c_{7} R_{a b c d} R^{a c} R^{b e} R_{e}^{d}+c_{8} R_{a b c d} R^{a c e f} R_{e}^{b} R_{f}^{d} \\
& +c_{9} R_{a b c d} R^{a c} R_{e f} R^{b e d f}+c_{10} R^{4}+c_{11} R^{2} R_{a b c d} R^{a b c d}+c_{12} R^{2} R_{a b} R^{a b} \\
& +c_{13} R_{a b c d} R^{a b e f} R_{e f g}^{c} R^{d g}+c_{14} R_{a b c d} R^{a e c f} R_{g e h f} R^{g b h d}
\end{aligned}
$$


with

$$
\begin{aligned}
& c_{1}=-(n-1)\left(n^{7}-3 n^{6}-29 n^{5}+170 n^{4}-349 n^{3}+348 n^{2}-180 n+36\right), \\
& c_{2}=-4(n-3)\left(2 n^{6}-20 n^{5}+65 n^{4}-81 n^{3}+13 n^{2}+45 n-18\right), \\
& c_{3}=-64(n-1)\left(3 n^{2}-8 n+3\right)\left(n^{2}-3 n+3\right) \\
& c_{4}=-\left(n^{8}-6 n^{7}+12 n^{6}-22 n^{5}+114 n^{4}-345 n^{3}+468 n^{2}-270 n+54\right), \\
& c_{5}=16(n-1)\left(10 n^{4}-51 n^{3}+93 n^{2}-72 n+18\right), \\
& c_{6}=--32(n-1)^{2}(n-3)^{2}\left(3 n^{2}-8 n+3\right), \\
& c_{7}=64(n-2)(n-1)^{2}\left(4 n^{3}-18 n^{2}+27 n-9\right), \\
& c_{8}=-96(n-1)(n-2)\left(2 n^{4}-7 n^{3}+4 n^{2}+6 n-3\right), \\
& c_{9}=16(n-1)^{3}\left(2 n^{4}-26 n^{3}+93 n^{2}-117 n+36\right) \\
& c_{10}=n^{5}-31 n^{4}+168 n^{3}-360 n^{2}+330 n-90, \\
& c_{11}=2\left(6 n^{6}-67 n^{5}+311 n^{4}-742 n^{3}+936 n^{2}-576 n+126\right), \\
& c_{12}=8\left(7 n^{5}-47 n^{4}+121 n^{3}-141 n^{2}+63 n-9\right), \\
& c_{13}=16 n(n-1)(n-2)(n-3)\left(3 n^{2}-8 n+3\right), \\
& c_{14}=8(n-1)\left(n^{7}-4 n^{6}-15 n^{5}+122 n^{4}-287 n^{3}+297 n^{2}-126 n+18\right) .
\end{aligned}
$$

In the action (1), $L(F)$ is the Lagrangian of power Maxwell invariant theory [16]

$$
L(F)=(-F)^{s}
$$

where $F=F_{\mu \nu} F^{\mu \nu}, F_{\mu \nu}=\partial_{\mu} A_{\nu}-\partial_{\nu} A_{\mu}$ is the electromagnetic field tensor and $A_{\mu}$ is the vector potential. One may note that in the limit $s=1, L(F)$ reduces to the standard Maxwell Lagrangian. Also, it is worth to mention that this Lagrangian is trivial for $s=0$.

In general, the variation of the action (11) with respect to the metric does not have a well-defined variational principle in the case of a manifold with boundary. This problem has been first noted gravity by Gibbons and Hawking for Einstein. They suggested that the following boundary term [11]

$$
I_{b}^{(1)}=\frac{1}{8 \pi} \int_{\partial \mathcal{M}} d^{n} x \sqrt{-\gamma} K
$$

makes the Einstein-Hilbert action well defined, where $\gamma_{a b}$ and $K$ are the induced metric and the trace of extrinsic curvature of the boundary $\partial \mathcal{M}$. This problem has been investigated 
for Lovelock gravity, and the boundary terms has been introduced in [12, 13]. Here, we are interested in the spacetimes with flat boundaries, $\hat{R}_{a b c d}(\gamma)=0$, and therefore the proper surface term for Gauss-Bonnet term is

$$
I_{b}^{(2)}=\frac{1}{8 \pi} \int_{\partial \mathcal{M}} d^{n} x \sqrt{-\gamma}\left\{\frac{2 \hat{\mu}_{2} l^{2}}{(n-2)(n-3)} J\right\},
$$

where $J$ is the trace of

$$
J_{a b}=\frac{1}{3}\left(2 K K_{a c} K_{b}^{c}+K_{c d} K^{c d} K_{a b}-2 K_{a c} K^{c d} K_{d b}-K^{2} K_{a b}\right)
$$

The surface terms for the curvature-cubed term of quasitopological gravity have been introduced in [14] as

$$
\begin{aligned}
I_{b}^{(3)}=\frac{1}{8 \pi} \int_{\partial \mathcal{M}} d^{n} x \sqrt{-\gamma} & \left\{\frac { 3 \hat { \mu _ { 3 } } l ^ { 4 } } { 5 n ( n - 2 ) ( n - 1 ) ^ { 2 } ( n - 5 ) } \left(n K^{5}-2 K^{3} K_{a b} K^{a b}\right.\right. \\
+ & 4(n-1) K_{a b} K^{a b} K_{c d} K_{e}^{d} K^{e c}- \\
& \left.\left.(5 n-6) K K_{a b}\left[n K^{a b} K^{c d} K_{c d}-(n-1) K^{a c} K^{b d} K_{c d}\right]\right)\right\} .
\end{aligned}
$$

Here, we are going to present a surface term that makes the action of quartic quasitopological gravity well defined. In order to do this, one should combine different seventh power of extrinsic curvature:

$$
\begin{aligned}
I_{b}^{(4)}=\frac{1}{8 \pi} \int_{\partial \mathcal{M}} d^{n} x \sqrt{-\gamma} & \frac{2 \hat{\mu}_{4} l^{6}}{7 n(n-1)(n-2)(n-7)\left(n^{2}-3 n+3\right)}\left\{\alpha_{1} K^{3} K^{a b} K_{a c} K_{b d} K^{c d}\right. \\
& +\alpha_{2} K^{2} K^{a b} K_{a b} K^{c d} K_{c}^{e} K_{d e}+\alpha_{3} K^{2} K^{a b} K_{a c} K_{b d} K^{c e} K_{e}^{d} \\
& +\alpha_{4} K K^{a b} K_{a b} K^{c d} K_{c}^{e} K_{d}^{f} K_{e f}+\alpha_{5} K K^{a b} K_{a}^{c} K_{b c} K^{d e} K_{d}^{f} K_{e f} \\
& \left.+\alpha_{6} K K^{a b} K_{a c} K_{b d} K^{c e} K^{d f} K_{e f}+\alpha_{7} K^{a b} K_{a}^{c} K_{b c} K^{d e} K_{d f} K_{e g} K^{f g}\right\}
\end{aligned}
$$

Using the $(n+1)$-dimensional static metric with a flat boundary

$$
d s^{2}=-g(\rho) d t^{2}+\frac{d \rho^{2}}{f(\rho)}+\rho^{2} \sum_{i=1}^{n-1} d \phi_{i}{ }^{2} .
$$

and the action (9), one may fix the unknown coefficients $\alpha_{i}$ 's such that the variation of $I_{b}^{(4)}$ 
removes all the normal derivatives of $\delta g_{\mu \nu}$ of quartic quasitopological term. One obtains

$$
\begin{aligned}
& \alpha_{1}=n^{2}(n-1), \\
& \alpha_{2}=2\left(n^{3}-4 n^{2}+3 n+3\right), \\
& \alpha_{3}=-6 n(n-1)^{2}, \\
& \alpha_{4}=-4 n\left(n^{2}-3 n+3\right)(n+2), \\
& \alpha_{5}=-2 n(n-3)\left(n^{2}-n-3\right), \\
& \alpha_{6}=2(n-1)\left(3 n^{3}-n^{2}-9 n+12\right), \\
& \alpha_{7}=24 .
\end{aligned}
$$

Thus, the boundary term that makes the action of quartic quasitopological gravity well

defined is the sum of all the boundary terms introduced above. That is $I_{b}=I_{b}^{(1)}+I_{b}^{(2)}+$ $I_{b}^{(3)}+I_{b}^{(4)}$.

In general, $I_{G}+I_{b}$ is divergent when evaluated on solutions, as is other thermodynamic quantities. Rather than eliminating these divergences by incorporating a reference term in the spacetime through the use of substraction method of Brown and York [17], one may add a new term $I_{c t}$, which is a functional of the boundary curvature invariants [18]. Since the boundary which we are interested in is flat, the proper counterterm is

$$
I_{c t}=-\frac{1}{8 \pi} \int_{\partial \mathcal{M}} d^{n} x \sqrt{-\gamma} \frac{(n-1)}{L_{e f f}},
$$

where $L_{e f f}$ is a scale length factor that depends on $l$ and the coupling constants of gravity and should reduce to $l$ in the absence of higher curvature terms.

\section{CHARGED BLACK BRANE SOLUTIONS}

Now, we introduce the charged black brane solutions of quasitopological gravity in the presence of the nonlinear Maxwell field with Lagrangian (44). Using the static metric (10) with $g(\rho)=N^{2}(\rho) f(\rho)$ and

$$
A_{\mu}=h(\rho) \delta_{\mu}^{0}
$$

for the vector potential, one can calculate the one dimensional action after integration by parts. One obtains the action per unit volume as

$$
I_{G}=\frac{(n-1)}{16 \pi l^{2}} \int d t d \rho\left[N(\rho)\left[\rho^{n}\left(1+\psi+\hat{\mu}_{2} \psi^{2}+\hat{\mu}_{3} \psi^{3}+\hat{\mu}_{4} \psi^{4}\right)\right]^{\prime}+\frac{2^{s} l^{2} \rho^{(n-1)} h^{2 s}}{(n-1) N(\rho)^{2 s-1}}\right],
$$


where $\psi=-l^{2} \rho^{-2} f(\rho)$ and the dimensionless parameters $\hat{\mu}_{2}, \hat{\mu}_{3}$ and $\hat{\mu}_{4}$ are defined as:

$$
\begin{gathered}
\hat{\mu}_{2} \equiv \frac{(n-2)(n-3)}{l^{2}} \mu_{2}, \quad \hat{\mu}_{3} \equiv \frac{(n-2)(n-5)\left(3 n^{2}-9 n+4\right)}{8(2 n-1) l^{4}} \mu_{3}, \\
\hat{\mu}_{4} \equiv \frac{n(n-1)(n-2)^{2}(n-3)(n-7)\left(n^{5}-15 n^{4}+72 n^{3}-156 n^{2}+150 n-42\right)}{l^{6}} \mu_{4},
\end{gathered}
$$

Varying the action (14) with respect to $\psi(r)$ yields

$$
\left(1+2 \hat{\mu}_{2} \psi+3 \hat{\mu}_{3} \psi^{2}+4 \hat{\mu}_{4} \psi^{3}\right) \frac{d N(\rho)}{d \rho}=0
$$

which shows that $N(\rho)$ should be a constant which we set equal to one. Variation with respect to $h(\rho)$ with $N(\rho)=1$ gives

$$
(2 s-1) \rho h^{\prime \prime}+(n-1) h^{\prime}=0,
$$

and therefore

$$
h(\rho)=\left\{\begin{array}{cc}
\frac{q}{2} \ln (\rho), & s=n / 2 \\
-q \rho^{-(n-2 s) /(2 s-1)}, & s \neq n / 2
\end{array}\right.
$$

where $q$ is an integration constant which is related to the charge parameter. We pause to give some comments on the allowed values of $s$. Equation (16) shows that the electromagnetic field equation is trivial for $s=1 / 2$. Also, in order to have a finite potential at infinity and therefore a finite energy for the charged solutions, $s$ should be in the range $1 / 2<s<n / 2$. Thus in the rest of the paper, we consider only the solutions for $1 / 2<s<n / 2$.

Varying the action with respect to $N(\rho)$ and substituting $N(\rho)=1$ gives

$$
\hat{\mu}_{4} \psi^{4}+\hat{\mu}_{3} \psi^{3}+\hat{\mu}_{2} \psi^{2}+\psi+\kappa=0
$$

where

$$
\kappa=1-\frac{m}{\rho^{n}}+\frac{2^{s} l^{2} q^{2 s}(n-2 s)^{2 s-1}}{(n-1)(2 s-1)^{(2 s-2)} \rho^{2 s(n-1) /(2 s-1)}},
$$

and $m$ is an integration constant which is related to the mass of the spacetime. The black brane solutions of (18), which reduces to black brane solution in Einstein gravity in the absence of higher curvature terms (the Einsteinian branch) is

$$
f(\rho)=\frac{\rho^{2}}{l^{2}}\left(\frac{\hat{\mu}_{3}}{4 \hat{\mu}_{4}}+\frac{1}{2} R-\frac{1}{2} E\right) .
$$


where

$$
\begin{aligned}
& R=\left(\frac{\hat{\mu}_{3}^{2}}{4 \hat{\mu}_{4}^{2}}-\frac{2 \hat{\mu}_{2}}{3 \hat{\mu}_{4}}+\left(\frac{D}{2}+\sqrt{\Delta}\right)^{1 / 3}+\left(\frac{D}{2}-\sqrt{\Delta}\right)^{1 / 3}\right)^{1 / 2}, \\
& E=\left(\frac{3 \hat{\mu}_{3}^{2}}{4 \hat{\mu}_{4}^{2}}-\frac{2 \hat{\mu}_{2}}{\hat{\mu}_{4}}-R^{2}-\frac{1}{4 R}\left[\frac{4 \hat{\mu}_{2} \hat{\mu}_{3}}{\hat{\mu}_{4}^{2}}-\frac{8}{\hat{\mu}_{4}}-\frac{\hat{\mu}_{3}^{3}}{\hat{\mu}_{4}^{3}}\right]\right)^{1 / 2}, \\
& \Delta=\frac{H^{3}}{27}+\frac{D^{2}}{4}, \quad H=\frac{3 \hat{\mu}_{3}-\hat{\mu}_{2}^{2}}{3 \hat{\mu}_{4}^{2}}-\frac{4 \kappa}{\hat{\mu}_{4}}, \\
& D=\frac{2}{27} \frac{\hat{\mu}_{2}^{3}}{\hat{\mu}_{4}^{3}}-\frac{1}{3}\left(\frac{\hat{\mu}_{3}}{\hat{\mu}_{4}^{2}}+8 \frac{\kappa}{\hat{\mu}_{4}}\right) \frac{\hat{\mu}_{2}}{\hat{\mu}_{4}}+\frac{\hat{\mu}_{3}^{2} \kappa}{\hat{\mu}_{4}^{3}}+\frac{1}{\hat{\mu}_{4}^{2}} .
\end{aligned}
$$

The metric function $f(\rho)$ for the uncharged solution $(q=0)$ is real in the whole range $0 \leq \rho<\infty$. But for charged solutions, one should restrict the spacetime to the region $\rho \geq r_{0}$, where $r_{0}$ is the largest real root of

$$
\begin{array}{r}
\frac{1}{4}\left[-\frac{2}{27} \frac{\hat{\mu}_{2}^{3}}{\hat{\mu}_{4}^{3}}+\frac{1}{3 \hat{\mu}_{4}}\left(\frac{\hat{\mu}_{3}}{\hat{\mu}_{4}^{2}}-\frac{4 \kappa_{0}}{\hat{\mu}_{4}}\right) \hat{\mu}_{2}-\frac{\hat{\mu}_{3}^{2} \kappa_{0}}{\hat{\mu}_{4}^{3}}+\frac{4 \hat{\mu}_{2} \kappa_{0}}{\hat{\mu}_{4}^{2}}-\frac{1}{\hat{\mu}_{4}^{2}}\right]^{2} \\
+\frac{1}{27}\left[\frac{\hat{\mu}_{3}}{\hat{\mu}_{4}^{2}}-\frac{4 \kappa_{0}}{\hat{\mu}_{4}}-\frac{\hat{\mu}_{2}^{2}}{3 \hat{\mu}_{4}^{2}}\right]^{3}=0
\end{array}
$$

where

$$
\kappa_{0}=1-\frac{m}{r_{0}^{n}}+\frac{2^{s} l^{2} q^{2 s}(n-2 s)^{2 s-1}}{(n-1)(2 s-1)^{(2 s-2)} r_{0}^{2 s(n-1) /(2 s-1)}},
$$

Performing the transformation

$$
r=\sqrt{\rho^{2}-r_{0}^{2}} \Rightarrow d \rho^{2}=\frac{r^{2}}{r^{2}+r_{0}^{2}} d r^{2}
$$

the metric becomes

$$
d s^{2}=-f(r) d t^{2}+\frac{r^{2} d r^{2}}{\left(r^{2}+r_{0}^{2}\right) f(r)}+\left(r^{2}+r_{0}^{2}\right) \sum_{i=1}^{n-1} d \phi_{i}^{2} .
$$

where now the functions $h(r)$ and $\kappa$ are

$$
\begin{aligned}
h(r) & =-q\left(r^{2}+r_{0}^{2}\right)^{(2 s-n) /(4 s-2)}, \\
\kappa & =1-\frac{m}{\left(r^{2}+r_{0}^{2}\right)^{n / 2}}+\frac{2^{s} l^{2} q^{2 s}(n-2 s)^{2 s-1}}{(n-1)(2 s-1)^{(2 s-2)}\left(r^{2}+r_{0}^{2}\right)^{s(n-1) /(2 s-1)}},
\end{aligned}
$$

The geometrical mass of black brane solutions in terms of the horizon radius, $r_{+}$, can be obtained as

$$
m=\left\{1+\frac{2^{s} l^{2} q^{2 s}(n-2 s)^{2 s-1}\left(r_{+}^{2}+r_{0}^{2}\right)^{s(1-n) /(2 s-1)}}{(n-1)(2 s-1)^{(2 s-2)}}\right\}\left(r_{+}^{2}+r_{0}^{2}\right)^{n / 2},
$$




\section{THERMODYNAMICS OF CHARGED BLACK BRANES}

The Hawking temperature for the black brane solution given in the last section can be calculated as:

$$
T=\frac{f^{\prime}\left(r_{+}\right)}{4 \pi} \sqrt{1+\frac{r_{0}^{2}}{r_{+}^{2}}}=\left\{\frac{n}{4 \pi l^{2}}-\frac{q^{2 s} 2^{s}(n-2 s)^{2 s}\left(r_{+}^{2}+r_{0}^{2}\right)^{s(1-n) /(2 s-1)}}{4 \pi(n-1)(2 s-1)^{2 s-1}}\right\} \sqrt{r_{+}^{2}+r_{0}^{2}},
$$

The electric potential $\Phi$, measured at infinity with respect to the horizon, is defined by [19]

$$
\Phi=\left.A_{\mu} \xi^{\mu}\right|_{r \rightarrow \infty}-\left.A_{\mu} \xi^{\mu}\right|_{r=r_{+}}
$$

where $\xi^{\mu}$ is the null generator of the horizon, $A_{\mu}$ is just the vector potentia.One obtains

$$
\Phi=\frac{q}{\left(r_{+}^{2}+r_{0}^{2}\right)^{(n-2 s) / 2(2 s-1)}},
$$

Using Gibbs free energy, we can calculate the entropy, electric charge and mass of this black hole. The Gibbs free energy in term of the on-shell action is $G=T I_{E}$, where $I_{E}$ is the Euclidean action and $T$ is the temperature [11].

The finite total action, $I_{E}=I_{g}+I_{b}+I_{c t}$ is finite, if $L_{e f f}$ of Eq. (12) is chosen as

$$
L_{e f f}=-\frac{210 \psi_{\infty}^{1 / 2}}{15 \hat{\mu_{4}} \psi_{\infty}^{4}+21 \hat{\mu}_{3} \psi_{\infty}^{3}+35 \hat{\mu}_{2} \psi_{\infty}^{2}-105 \psi_{\infty}-105} l,
$$

where $\psi_{\infty}$ is the limit of $\psi$ at infinity for our solution given in Eq. (19). The finite action per unit volume can be calculated as

$$
I_{E}=-\frac{\beta}{16 \pi l^{2}}\left\{\left(r_{+}^{2}+r_{0}^{2}\right)^{n / 2}+\frac{2^{s} l^{2} q^{2 s}(n-2 s)^{2 s-1}\left(r_{+}^{2}+r_{0}^{2}\right)^{(2 s-n) / 2(2 s-1)}}{(2 s-1)^{2 s-2}(n-1)}\right\},
$$

Having the total finite action, one can obtain the Gibbs free energy per unit volume as

$$
G(T, \Phi)=-\frac{1}{16 \pi l^{2}}\left\{\left(r_{+}^{2}+r_{0}^{2}\right)^{n / 2}+\frac{2^{s} l^{2}(n-2 s)^{2 s-1} \Phi^{2 s}\left(r_{+}^{2}+r_{0}^{2}\right)^{(n-2 s) / 2}}{(2 s-1)^{2 s-2}(n-1)}\right\},
$$

where the implicit dependence of $r_{+}^{2}+r_{0}^{2} \equiv \rho_{+}{ }^{2}$ in terms of the intensive quantities $T$ and $\Phi$ is

$$
n(n-1) \rho_{+}^{2}-4 \pi l^{2}(n-1) T \rho_{+}-2^{s}(n-2 s)^{2 s}(2 s-1)^{1-2 s} l^{2} \Phi^{2 s} \rho_{+}^{2(1-s)}=0 .
$$

It is notable that for $s=1$, the above equation may be solved for $\rho_{+}$, and therefore one can find the explicit form of $\rho_{+}$in terms of $T$ and $\Phi$ [14]. Here, one can calculate $\left(\partial T / \partial \rho_{+}\right)_{\Phi}$ 
and $\left(\partial \Phi / \partial \rho_{+}\right)_{T}$ and the entropy and charge per unit volume of charged black brane may be obtained by using the well-known equations

$$
\begin{aligned}
& S=-\left(\frac{\partial G}{\partial T}\right)_{\Phi}=-\left(\frac{\partial G}{\partial \rho_{+}}\right)_{\Phi}\left(\frac{\partial T}{\partial \rho_{+}}\right)_{\Phi}^{-1}=\frac{1}{4}\left(r_{+}^{2}+r_{0}^{2}\right)^{(n-1) / 2} \\
& Q=-\left(\frac{\partial G}{\partial \Phi}\right)_{T}=-\left(\frac{\partial G}{\partial \Phi}\right)_{T}-\left(\frac{\partial G}{\partial \rho_{+}}\right)_{T}\left(\frac{\partial \Phi}{\partial \rho_{+}}\right)_{T}^{-1}=\frac{2^{s} s(n-2 s)^{2 s-1} q^{2 s-1}}{8 \pi(2 s-1)^{2 s-1}}
\end{aligned}
$$

which show that they are in agreement with the entropy density calculated in Ref. [7] and the charge density that may be obtained by use of Gauss theorem, respectively.

In order to check the first law of thermodynamics of the black brane, one may write the energy density as a function of extensive parameters, $S$ and $Q$ as

$$
\begin{aligned}
M(S, Q) & =G+T S+\Phi Q=\frac{n-1}{16 \pi} m \\
& =\frac{n-1}{16 \pi}\left[(4 S)^{2 s /(2 s-1)}+\frac{(8 \pi / s)^{2 s /(2 s-1)} l^{2}(2 s-1)^{2}}{2^{s /(2 s-1)}(n-1)(n-2 s)} Q^{2 s /(2 s-1)}\right](4 S)^{(2 s-n) /(n-1)} .
\end{aligned}
$$

The first law of thermodynamics, $d M=T d S+\Phi d Q$, is satisfied. This is due to the fact that the intensive quantities

$$
T=\left(\frac{\partial M}{\partial S}\right)_{Q}, \quad \Phi=\left(\frac{\partial M}{\partial Q}\right)_{S} .
$$

are in agreement with those given in Eqs. (31) and (33).

Also, it is worth to mention that the energy per unit volume of the black brane in terms of the thermodynamics quantities can be written as

$$
M=\frac{n-1}{n}(T S+Q \Phi)
$$

\section{THERMODYNAMICS OF CHARGED ROTATING BLACK BRANES}

Here we consider the metric of $(n+1)$-dimensional rotating spacetime with flat horizon and $k$ rotation parameters, which may be written as [20]

$$
\begin{aligned}
d s^{2}= & -f(r)\left(\Xi d t-\sum_{i=1}^{k} a_{i} d \phi_{i}\right)^{2}+\frac{r^{2}+r_{0}^{2}}{l^{4}} \sum_{i=1}^{k}\left(a_{i} d t-\Xi l^{2} d \phi_{i}\right)^{2} \\
& +\frac{r^{2} d r^{2}}{\left(r^{2}+r_{0}^{2}\right) f(r)}-\frac{r^{2}+r_{0}^{2}}{l^{2}} \sum_{i<j}^{k}\left(a_{i} d \phi_{j}-a_{j} d \phi_{i} \phi_{i}\right)^{2}+\left(r^{2}+r_{0}^{2}\right) \sum_{i=k+1}^{n-1} d \phi_{i}{ }^{2},
\end{aligned}
$$


where the angular coordinates are in the range $0 \leq \phi_{i}<2 \pi, \Xi=\sqrt{1+\mathbf{a}^{2} / l^{2}}$ with $\mathbf{a}^{2}=$ $\sum_{i=1}^{k} a_{i}^{2}$ and $f(r)$ is the same as the metric function in static case. One should note that the maximum number of rotation parameter in $(n+1)$ dimensions is $k=[n / 2]$, where $[x]$ is the integer part of $x$. Although the metrics (27) and (41) can be mapped into each other locally by the transformation

$$
t^{\prime}=\Xi t-\sum_{i=1}^{k} a_{i} \phi_{i}, \quad \phi_{i}^{\prime}=\frac{a_{i}}{l^{2}} t-\Xi \phi_{i},
$$

they cannot mapped into each other globally. This is due to the periodic nature of the coordinates $\phi_{i}, i=1 \ldots k[21]$.

Now, we investigate the thermodynamics of rotating charged black branes. The gauge potential for this solution is given by

$$
A_{\mu}=h(r)\left(\Xi d t-\sum_{i=1}^{k} a_{i} d \phi_{i}\right),
$$

where $h(r)$ is given in Eq. (28). The temperature of the Killing horizon is given by

$$
T=\frac{1}{2 \pi}\left(-\frac{1}{2} \nabla_{b} \xi_{a} \nabla^{b} \xi^{a}\right)_{r=r_{+}}^{1 / 2},
$$

where $\xi$ is the combination of Killing vectors

$$
\xi=\partial_{t}+\sum_{i=1}^{k} \Omega_{i} \partial_{\phi_{i}}
$$

and $\Omega_{i}$ is the angular velocity of the horizon given as

$$
\Omega_{i}=-\left[\frac{g_{t \phi_{i}}}{g_{\phi_{i} \phi_{i}}}\right]_{r=r_{+}}=\frac{a_{i}}{\Xi l^{2}} .
$$

Using Eqs. (43)-(45), one obtains

$$
T=\frac{1}{\Xi}\left\{\frac{n}{4 \pi l^{2}}-\frac{q^{2 s} 2^{s}(2 s-1)[(n-2 s) /(2 s-1)]^{2 s}\left(r_{+}^{2}+r_{0}^{2}\right)^{s(1-n) /(2 s-1)}}{4 \pi(n-1)}\right\} \sqrt{r_{+}^{2}+r_{0}^{2}} .
$$

The electric potential $\Phi$ measured at infinity with respect to the horizon is

$$
\Phi=\frac{1}{\Xi} \frac{q}{\left(r_{+}^{2}+r_{0}^{2}\right)^{(n-2 s) / 2(2 s-1)}} .
$$

The finite action per unit volume can be calculated through the use of the counterterm method as

$$
I_{E}=-\frac{\beta}{16 \pi l^{2}}\left\{\left(r_{+}^{2}+r_{0}^{2}\right)^{n / 2}+\frac{2^{s} l^{2} q^{2 s}(n-2 s)^{2 s-1}\left(r_{+}^{2}+r_{0}^{2}\right)^{(2 s-n) / 2(2 s-1)}}{(2 s-1)^{2 s-2}(n-1)\left(1-l^{2} \Omega^{2}\right)}\right\} .
$$


Using Eqs. (45), (46) and (47), the Gibbs free energy per unit volume in terms of the intensive quantities $T, \Phi$ and $\Omega_{i}$ 's may be written as

$$
G(T, \Phi, \boldsymbol{\Omega})=-\frac{1}{16 \pi l^{2}}\left\{\left(r_{+}^{2}+r_{0}^{2}\right)^{n / 2}+\frac{2^{s} l^{2}(n-2 s)^{2 s-1} \Phi^{2 s}\left(r_{+}^{2}+r_{0}^{2}\right)^{(n-2 s) / 2}}{\left(1-l^{2} \Omega^{2}\right)(2 s-1)^{2 s-2}(n-1)}\right\}
$$

where $\Omega^{2}=\sum_{i=1}^{k} \Omega_{i}^{2}$. Now using the well-known formulas of entropy, charge, energy per volume of rotating charged black brane in term of the Gibbs free energy and the relation between $\eta_{+}, T$ and $S$, one obtains

$$
\begin{gathered}
S=-\left(\frac{\partial G}{\partial T}\right)_{\Phi, \Omega_{i}}=\frac{\Xi}{4}\left(r_{+}^{2}+r_{0}^{2}\right)^{(n-1) / 2}, \\
Q=-\left(\frac{\partial G}{\partial \Phi}\right)_{T, \Omega_{i}}=\Xi \frac{[(n-2 s) /(2 s-1)]^{2 s-1} 2^{s} s q^{2 s-1}}{8 \pi} . \\
J_{i}=-\left(\frac{\partial G}{\partial \Omega_{i}}\right)_{T, \Phi}=\frac{n}{16 \pi} \Xi m a_{i} .
\end{gathered}
$$

The energy density per unit volume may be written as

$$
M=G+T S+\Phi Q+\sum_{i=1}^{k} \Omega_{i} J_{i}=\frac{1}{16 \pi}\left(n \Xi^{2}-1\right) m .
$$

Again, one may confirm the first law of thermodynamics by computing the angular velocities, temperature and potential

$$
\Omega_{i}=\left(\frac{\partial M}{\partial J_{i}}\right)_{S, Q}, \quad T=\left(\frac{\partial M}{\partial S}\right)_{Q, \mathbf{J}}, \quad \Phi=\left(\frac{\partial M}{\partial Q}\right)_{S, \mathbf{J}},
$$

and compare them with $\Omega_{i}, T$ and $\Phi$ given in Eqs. (45), (46) and (47), respectively.

\section{CONCLUDING REMARKS}

In this paper, our first aim was to introduce a surface term that makes the action of quartic quasitopological gravity well defined. This was done by introducing seven terms of order seven in extrinsic curvature of the boundary with suitable coefficients in such a way that cancel the derivative of the variation of the metric normal to the flat boundary. Although, we have used this boundary term in order to calculate the on-shell action of black hole solution of the quasitopological gravity, one may use it in Hamiltonian formalism of quasitopological gravity. Having the nonlinear terms in Riemann tensor in the action of 
gravity, we assumed to have nonlinear terms in the invariant quantity of the electromagnetic field tensor too. That is, we considered the quartic quasitopological gravity in the presence of

nonlinear electromagnetic field introduced in [16], and introduced the black brane solutions of this theory. These solutions may be interpreted as black brane solutions with two inner and outer event horizons or an extreme black brane depending on the value of charge parameter.

In order to investigate the thermodynamics of these charged solutions, one needs the finite on-shell action of the solutions. But, as in the case of Einstein solutions, the action and the conserved quantities of the solutions of quasitopological gravity are not finite. We used the counterterm method to calculate the finite action. Using the definition of Gibbs free energy for the solutions, we computed the thermodynamic quantities $S, Q$ and the energy of the solutions per unit volume. We found that the thermodynamic and conserved quantities of black branes of quasitopological gravity with flat horizon are independent of the the GaussBonnet and quasi topological coefficients $\left(\mu_{2}, \mu_{3}\right.$ and $\left.\mu_{4}\right)$. We obtained the mass as a function of the extensive parameters $S$ and $Q$ and showed that the conserved and thermodynamic quantities satisfy the first law of thermodynamic. Finally we generalized the solutions to the rotating solutions and obtained the conserved and thermodynamic quantities of these rotating charged solutions. The surface terms of quartic quasitopological gravity introduced here may be used in the investigation of the thermodynamics of asymptotic Lifshitz black branes, which will be given elsewhere.

\section{Acknowledgments}

This work has been supported financially by Center for Excellence in Astronomy \& Astrophysics (CEAA - RIAAM) under research project No. 1/3232.

[1] J. M. Maldacena, Adv. Theor. Math. Phys. 2, 231 (1998) [Int. J. Theor. Phys. 38, 1113 (1999)]; S. S. Gubser, I. R. Klebanov and A. M. Polyakov, Phys. Lett. B 428, 105 (1998); E. Witten, Adv. Theor. Math. Phys. 2, 253-291 (1998).

[2] A. Buchel, J. T. Liu and A. O. Starinets, Nucl. Phys. B 707, 56 (2005); Y. Kats and P. Petrov, J. High Energy Phys. 01 (2009) 044.

[3] D. Lovelock, J. Math. Phys. 12, 498 (1971). 
[4] R. C. Myers, M. F. Paulos and A. Sinha, Phys. Rev. D 79, 041901 (2009); A. Buchel, R. C. Myers, M. F. Paulos and A. Sinha, Phys. Lett. B 669, 364 (2008); A. Buchel, R. C. Myers and A. Sinha, J. High Energy Phys. 03 (2009) 084; A. Sinha and R. C. Myers, Nucl. Phys. A 830, 295C (2009).

[5] D. M. Hofman and J. Maldacena, J. High Energy Phys. 05 (2008) 012; D. M. Hofman, Nucl. Phys. B 823, 174 (2009).

[6] R. C. Myers and B. Robinson, J. High Energy Phys. 08 (2010) 067.

[7] M. H. Dehghani, et al. Phys. Rev. D 85, 104009 (2012).

[8] R. C. Myers, M. F. Paulos, A. Sinha, J. High Energy Phys. 08 (2010) 035.

[9] X. -M. Kuang, W. -J. Li, Y. Ling, J. High Energy Phys. 12 (2010) 069; J. -P. Wu, J. High Energy Phys. 07 (2011) 106; X. -M. Kuang, W. -J. Li, Y. Ling, arXiv:1106.0784 [hep-th]; K. B. Fadafan, arXiv:1102.2289 [hep-th].

[10] W. G. Brenna, M. H. Dehghani, and R. B. Mann, Phys. Rev. D 84, 024012 (2011).

[11] G. W. Gibbons, S. W. Hawking, Phys. Rev. D 15, 2752-2756 (1977).

[12] R. C. Myers, Phys. Rev. D 36, 392 (1987); S. C. Davis, ibid. 67, 024030 (2003).

[13] M. H. Dehghani and R. B. Mann, Phys. Rev. D 73, 104003 (2006); M. H. Dehghani, N. Bostani and A. Sheykhi, ibid. 73, 104013 (2006).

[14] M. H. Dehghani and M. H. Vahidinia, Phys. Rev. D 84, 084044 (2011).

[15] J. W. York, Jr., Phys. Rev. Lett. 28, 1082 (1972).

[16] M. Hassaine and C. Martinez, Phys. Rev. D 75, 027502 (2007); M. Hassaine and C. Martinez, Class. Quant. Grav. 25, 195023 (2008); H. Maeda, M. Hassaine and C. Martinez, Phys. Rev. D 79, 044012 (2009); S. Habib Mazharimousavi and M. Halilsoy, Phys. Lett. B 681, 190 (2009); S. H. Hendi, Phys. Lett. B 678, 438 (2009); S. H. Hendi, Class. Quant. Grav. 26, 225014 (2009).

[17] J. D. Brown and J. W. York, Phys. Rev. D 47, 1407 (1993); J. D. Brown, J. Creighton and R. B. Mann, Phys. Rev. D 50, 6394 (1994); I. S. Booth and R. B. Mann, Phys. Rev. D 59, 064021 (1999).

[18] M. Henningson and K. Skenderis, J. High Energy Phys. 07 (1998) 023; Fortsch. Phys. 48, 125 (2000); S. Nojiri, S. D. Odintsov, Phys. Lett B 444, 92 (1998); S. Hyun, W. T. Kim and J. Lee, Phys. Rev. D 59, 084020 (1999); V. Balasubramanian and P. Kraus, Commun. Math. Phys. 208, 413 (1999). 
[19] M. Cvetic and S. S. Gubser, J. High Energy Phys. 04, 024 (1999); M. M. Caldarelli, G. Cognola and D. Klemm, Class. Quantum Grav. 17, 399 (2000).

[20] A. M. Awad, Class. Quant. Grav. 20, 2827 (2003).

[21] J. Stachel, Phys. Rev. D 26, 1281 (1982). 